\title{
Satisfacción laboral y salud: Un análisis de efectos directos e indirectos en gerentes argentinos ${ }^{*}$
}

\author{
Job Satisfaction and Health: An Examination of Direct and Indirect Effects Among Argentinian Managers \\ Satisfação Laboral e aúde: uma análise dos efeitos diretos e indiretos em gerentes argentinos
}

Lucas Pujol-Cols ${ }^{a}$

Consejo Nacional de Investigaciones Cientificas y Técnicas

(CONICET), Argentina

Universidad Católica del Maule, Chile

lucaspujolcols@gmail.com

ORCID: http://orcid.org/0000-0002-8754-3416

Marina Foutel

Universidad Nacional de Mar del Plata, Argentina

ORCID: http://orcid.org/0000-0002-7208-355X
DOI: https://doi.org/10.11144/Javeriana.cao32-59.slsaed Redalyc: http://www.redalyc.org/articulo.oa? $\mathrm{id}=$ cuadernoss 20561244003

Fecha de recepción: 23 Septiembre 2018

Fecha de aprobación: 11 Octubre 2019 Fecha de publicación: 15 Diciembre 2019

\section{Resumen:}

Este estudio examina la prevalencia de un conjunto de síntomas relacionados con la salud física y mental en una muestra de 124 gerentes argentinos. Subsecuentemente, analiza los efectos directos e indirectos de la satisfacción laboral sobre la salud. Los análisis de prevalencia revelan que más del 75\% de los directivos suelen sentirse estresados, cansados, tensos, nerviosos, desanimados y/o agotados mentalmente. Además, entre el 10\% y el 35\% suelen experimentar dolores de espalda, problemas de sueño, dolores de cabeza, indigestión ácida, tensión ocular, fatiga, trastornos estomacales, erupciones cutáneas y/o diarrea. El análisis de ecuaciones estructurales muestra que la satisfacción laboral ejerce un efecto indirecto sobre la salud física a través de la salud mental.

Código JEL: C01, I00, M12

Palabras clave: satisfacción laboral, salud mental, salud física, gerentes.

\section{Abstract:}

This study examines the prevalence of a set of mental and physical health symptoms in a sample of 124 Argentinian managers. Subsequently, it analyzes the direct and indirect effects of job satisfaction on health. The results reveal that above $75 \%$ of the participants feel stressed-out, tired, tense, nervous, depressed, and/or mentally exhausted on a regular basis. Furthermore, between $10 \%$ and $35 \%$ of the participants report experiencing backaches, sleep difficulties, headaches, indigestion, eyestrain, fatigue, stomachaches, rashes, and/or diarrhea. Results from the structural equation modeling show that job satisfaction exerts an indirect effect on physical health through mental health.

JEL Codes: C01, I00, M12

Keywords: Job satisfaction, mental health, physical health, managers.

\section{Resumo:}

Este estudo tem como objetivo examinar a prevalência de um conjunto de sintomas relacionados à saúde física e mental em uma amostra de 124 gestores argentinos. Posteriormente, são analisados os efeitos diretos e indiretos da satisfação no trabalho sobre a saúde. As análises de prevalência revelam que mais de $75 \%$ dos gerentes tendem a se sentir estressados, cansados, tensos, nervosos, desanimados e / ou mentalmente exaustos. Além disso, entre 10\% e 35\% geralmente apresentam dores nas costas, problemas de sono, dores de cabeça, indigestão ácida, tensão ocular, fadiga, distúrbios estomacais, erupções cutâneas e / ou diarreia. A análise de equações estruturais mostra que a satisfação no trabalho tem um efeito indireto sobre a saúde física através da saúde mental.

Código JEL: C01, I00, M12

Palavras-chave: satisfação no trabalho, saúde mental, saúde física, gestores.

\section{Notas de autor}




\section{INTRODUCCIÓN}

Los profundos cambios que se han producido en el mundo del trabajo en las últimas décadas se han traducido en nuevas demandas y desafíos para los trabajadores alrededor del mundo, así como en mayores niveles de distrés ocupacional (Siegrist, 2016; Salanova, Martínez, \& Llorens, 2014; Unda, Uribe, Jurado, García, Tovalín \& Juárez, 2016). Este escenario laboral caracterizado por niveles crecientes de exposición a riesgos psicosociales ha incrementado la preocupación de funcionarios, académicos, directivos y representantes sindicales por mejorar las condiciones de trabajo en las organizaciones, con miras a preservar la salud física y mental del capital humano (Pujol-Cols \& Arraigada, 2017). El estudio del bienestar en el trabajo y, en particular, de las condiciones individuales y organizacionales que coadyuvan a preservarlo, han constituido el principal objeto de estudio de la psicología organizacional positiva (Seligman \& Csikszentmihalyi, 2000), definida como el estudio científico y la gestión del funcionamiento óptimo de las personas y grupos en las organizaciones (Schaufeli, Salanova, González-Roma \& Bakker, 2002).

Según un informe emitido por Forbes (2017), la profesión gerencial constituye una de las diez ocupaciones más estresantes a nivel mundial. En efecto, numerosas investigaciones reconocen que los directivos enfrentan elevadas demandas cognitivas en su trabajo. Esto se debe no sólo a la complejidad de sus funciones (Dabos \& Rivero, 2012; Ford \& Collinson, 2011), sino también a la responsabilidad que implica resolver problemas difíciles y adoptar decisiones efectivas en contextos inciertos, turbulentos y dinámicos (Sprinkle, 2003; Luft \& Shields, 2003; Birnberg, Luft \& Shields, 2007). Al mismo tiempo, los directivos enfrentan elevadas demandas emocionales, debiendo no sólo regular o suprimir algunas de sus emociones para ser efectivos en su rol, sino también observar comportamientos social y organizacionalmente deseables durante su interacción con múltiples agentes de interés (Brotheridge \& Grandey, 2002; Gardner \& Stough, 2002). Por otra parte, la sobrecarga de trabajo, así como el agotamiento emocional que suele derivarse de ésta, los vuelve más proclives a experimentar interferencias o incompatibilidades entre las demandas del trabajo y las de la vida personal (Montgomery, Peeters, Schaufeli, \& Ouden, 2003).

Ante dicho escenario se deduce que los gerentes representan un sector ocupacional especialmente vulnerable a experimentar distrés, entendido como la respuesta fisiológica o emocional del individuo ante una demanda que es percibida por aquel como dañina (Ridner, 2004). En efecto, los directivos resultan más proclives que otros grupos a sentir que sus recursos personales se derrumban en su intento por enfrentar las elevadas demandas que emergen de su profesión (Christie \& Barling, 2009). Esto, a su vez, incrementa su propensión a experimentar numerosos estados negativos, como agotamiento emocional, burnout y pérdidas de bienestar (Bowling, Alarcon, Bragg \& Hartman, 2015; Nixon, Mazzola, Bauer, Krueger \& Spector, 2011; Pindek, Arvan \& Spector, 2018). Sin embargo, en el contexto argentino, no se identificaron investigaciones focalizadas en la naturaleza psicosocial del trabajo directivo, así como en sus efectos sobre el bienestar, las actitudes y los comportamientos de este grupo profesional, al encontrarse mayormente concentradas en los empleados.

Con la intención de impulsar futuros estudios en la temática, así como el desarrollo de políticas que contribuyan a preservar la salud ocupacional de los directivos, este artículo persigue dos objetivos. Por un lado, examina la prevalencia de un conjunto de síntomas relacionados con la salud física y mental en una muestra de gerentes argentinos. Por el otro, analiza los efectos directos e indirectos de la satisfacción laboral sobre la salud a través de ecuaciones estructurales. Entender los mecanismos que contribuyen a que los gerentes experimenten estados más saludables en su trabajo resulta fundamental por dos motivos. En primer lugar, para impulsar el diseño de políticas y estrategias de gestión que coadyuven a preservar la integridad física y psicológica de estos profesionales. En segundo lugar, por las implicaciones que la salud tiene sobre la efectividad organizacional, al asumir los gerentes un rol crítico en la viabilidad de las organizaciones y el cumplimiento de la misión organizativa, así como también en las decisiones y prácticas que afectan la calidad de vida del capital humano bajo su dirección (Hosie \& Sevastos, 2009). 


\section{LA PROFESIÓN GERENCIAL DESDE UNA PERSPECTIVA PSICOSOCIAL}

Numerosas investigaciones señalan que el trabajo gerencial suele ser muy variado, fragmentado y complejo. Es un trabajo variado pues implica una gran cantidad de tareas diversas, como reuniones, llamadas telefónicas, la presencia en eventos, etc. (Hall, 2010; Braithwaite, Westbrook, \& Mallock, 2007). Es también una labor fragmentada, ya que el ejercicio de cada tarea consume relativamente poco tiempo o se ve afectado por quiebres o interrupciones que obligan al individuo a concentrarse rápidamente en otra actividad (Mark, Gonzalez, \& Harris, 2005). Finalmente, es un trabajo complejo porque requiere la inversión de un elevado volumen de energía cognitiva, así como la aplicación de conocimientos y talentos diversos (Dabos \& Rivero, 2012). Además de estas demandas de tipo cognitivo, los directivos enfrentan elevadas demandas emocionales. Por un lado, deben regular o suprimir algunas de sus emociones para ser efectivos en el ejercicio de su rol, lo que incrementa, a su vez, la probabilidad de experimentar disonancia emocional (Lewig \& Dollard, 2003). Por el otro, deben transmitir emociones positivas (p. ej., entusiasmo, optimismo o empatía) y observar comportamientos social y organizacionalmente deseables durante su interacción con múltiples agentes de interés, tales como clientes, subordinados, colegas, supervisores, propietarios, funcionarios, proveedores, representantes sindicales, miembros de la comunidad y entidades de control y acreditación (Braithwaite et al., 2007; Brotheridge \& Grandey, 2002; Downey, Papageorgiou \& Stough, 2006; Gardner \& Stough, 2001).

Por otra parte, Ford y Collinson (2011) señalan que los directivos suelen trabajar muchas más horas que las acordadas inicialmente en sus contratos de empleo, y se asume que deben estar disponibles en todo momento para resolver asuntos de trabajo. Esto no sólo tiende a devenir en niveles elevados de sobrecarga mental y agotamiento (O’Neill \& Xiao, 2010), sino que además conduce a interferencias entre el trabajo y la vida personal, disminuyendo aún más la capacidad de los directivos para responder eficazmente a las demandas provenientes de ambos dominios (Lucia-Casademunt, Ariza-Montes \& Morales-Gutiérrez, 2013; Montgomery et al., 2003). Así, como resultado de relegar su vida personal a un lugar residual, los directivos suelen contar con menos tiempo promedio para recuperarse cognitiva y emocionalmente del distrés diario y, de este modo, encontrarse listos para afrontar una nueva jornada de trabajo. Por otro lado, la literatura también señala que, como resultado de las elevadas expectativas impuestas sobre el rol del directivo y su grado de efectividad pretendido, estos suelen experimentar mayor inseguridad laboral que otros grupos, siendo más proclives al despido en caso de errores (Collinson \& Collinson, 1997).

Siguiendo a Bakker y Demerouti (2014), los individuos insertos en profesiones altamente demandantes deberían recibir elevados recursos laborales, es decir, aquellos factores físicos, psicológicos, organizacionales o sociales del trabajo que son relevantes para lograr los objetivos, son funcionales para reducir las demandas laborales y estimulan el desarrollo de los empleados. Sin embargo, algunas investigaciones demuestran que los directivos suelen sentir que el apoyo social y el reconocimiento que reciben de sus supervisores, colegas y subordinados suele ser insuficiente (Lucia-Casademunt et al., 2013; Manshor, Fontaine, \& Siong Choy, 2003). Frente a este escenario ocupacional se deduce que los gerentes representan un sector ocupacional especialmente vulnerable a experimentar distrés y pérdidas de bienestar (Hosie \& Sevastos, 2009). Por ejemplo, Rout (1999) señala que los directivos suelen experimentar menor satisfacción laboral que el resto de los empleados, al sentir que el apoyo que reciben de los propietarios, así como de sus colegas, subordinados y supervisores, es muy limitado. De modo similar, Mohr y Wolfram (2010) reportan que los directivos tienden a experimentar irritación emocional cuando sienten que sus tareas son excesivamente dinámicas e inciertas, fenómeno que se ve intensificado aún más cuando el apoyo social que reciben es percibido como insuficiente. Por otro lado, O’Neill y Xiao (2010) demuestran que aquellos directivos que experimentan mayores demandas en su trabajo resultan más proclives a sentirse emocionalmente agotados. 
Más recientemente, Hentrich, Sosnowsky-Waschek, Gregersen y Petermann (2017) reportan que los gerentes suelen experimentar depresión cuando sienten que las demandas cuantitativas y emocionales de su trabajo son demasiado intensas.

Los estudios sobre la naturaleza psicosocial del trabajo directivo y sus efectos sobre el bienestar son más limitados en la literatura iberoamericana. A modo de ejemplo, en una investigación realizada en el contexto mexicano, Mercado Salgado y Salgado Mejía (2008) reportan que los directivos participantes trabajan en promedio 53 horas a la semana, indicando además que los principales factores del trabajo que afectan su bienestar están dados por las grandes responsabilidades, las dificultades involucradas en la resolución de los problemas, la falta de un reconocimiento que compense adecuadamente los esfuerzos y el conflicto trabajofamilia. En cuanto al contexto argentino en particular, no fue posible identificar ninguna investigación previa publicada que haya examinado los efectos del trabajo sobre el bienestar en este grupo ocupacional.

\section{La satisfacción laboral y sus efectos sobre la salud}

La salud representa mucho más que la ausencia de enfermedad. En efecto, debe ser entendida en sentido amplio como "un estado de bienestar total que incluye el bienestar físico, mental y social" (Salanova, Martínez \& Llorens, 2014, p. 23). La psicología organizacional positiva, en particular, consiste en el estudio científico del funcionamiento óptimo de la salud de los individuos y grupos en contextos organizacionales. Tiene como propósito desarrollar organizaciones saludables que permitan mejorar la calidad de vida laboral y potenciar el bienestar psicosocial en el trabajo (Pujol-Cols \& Arraigada, 2018). A diferencia de otros enfoques mayormente centrados en los trastornos, las aflicciones y las enfermedades asociadas al trabajo (por ejemplo, el burnout), la psicología organizacional positiva focaliza su atención en el estudio de los mecanismos individuales, grupales y organizacionales que contribuyen a la experimentación de estados positivos, como la salud y el bienestar.

Numerosas investigaciones han demostrado que el grado en que los individuos experimentan actitudes positivas hacia el trabajo, como por ejemplo satisfacción laboral, no sólo tiene fuertes implicaciones para la efectividad organizacional (p. ej., Diestel, Wegge \& Schmidt, 2014; Flickinger, Allscher \& Fiedler, 2016; Tziner, Waismal-Manor, Vardi \& Brodman, 2008; Ziegler, Hagen \& Diehl, 2012), sino también para la conservación de la salud (p. ej., Grant, Wardle \& Steptoe, 2009; Tsaousis, Nikolaou, Serdaris \& Judge, 2007). En este sentido, existe evidencia que señala que el trabajo ejerce un rol protector en la salud de los individuos cuando logra brindarles las condiciones necesarias para que satisfagan sus necesidades personales (Satuf, Monteiro, Pereira, Esgalhado, Marina Afonso \& Loureiro, 2018). A modo de ejemplo, en un meta-análisis realizado sobre 485 investigaciones publicadas en PsycINFO, PubMed, Social Sciences Citation Index, Arts \& Humanities Citation Index y ERIC, Faragher, Cass y Cooper (2013) reportan correlaciones moderadas y estadísticamente significativas entre la satisfacción laboral y numerosas variables vinculadas con la salud mental, tales como el distrés $(\mathrm{r}=-0,31)$, la depresión $(\mathrm{r}=-0,37)$, la ansiedad $(\mathrm{r}=-0,35)$, el bienestar psicológico $(\mathrm{r}=0,32)$ y el burnout $(\mathrm{r}=-0,41)$. Sin embargo, los autores señalan que la relación entre la satisfacción laboral y la salud física $(\mathrm{r}=0,24)$ suele ser más débil que la identificada entre la satisfacción laboral y la salud mental. Esto ocurre especialmente en el caso de las sintomatologías físicas más graves, como las enfermedades cardiovasculares $(\mathrm{r}=-0,11)$ y los desórdenes musculo-esqueléticos $(\mathrm{r}=-0,08)$.

Una explicación tentativa a las relaciones entre la satisfacción laboral, la salud mental y la salud física podría hallarse en la denominada hipótesis del derrame (spillover hypothesis). Desde esta perspectiva, el trabajo representa una dimensión central en la vida de los individuos, que les confiere un sentido profundo de propósito y significado, siendo al mismo tiempo altamente instrumental para la satisfacción de un abanico de necesidades superiores, como el crecimiento personal (Weir, 2013). Asimismo, puesto que los empleados más satisfechos tienden a poseer mayores niveles de work engagement (Pujol-Cols \& LazzaroSalazar, 2018), también resultan más proclives a sentirse plenos durante el ejercicio de las tareas con las que se hallan psicológicamente implicados, experimentando, en consecuencia, emociones más positivas 
durante el ejercicio de su rol, como placer, alegría, entusiasmo, felicidad y disfrute (Bakker \& Demerouti, 2014). Por estas razones, la satisfacción laboral constituye un determinante fundamental del bienestar subjetivo (Diener \& Tay, 2017) y la satisfacción con la vida (Bowling, Eschleman \& Wang, 2010; Unanue, Gómez, Cortez, Oyanedel \& Mendiburo-Seguel, 2017), encontrándose, como resultado, positivamente relacionada con la experimentación de estados mentales más saludables (Bakker \& Demerouti, 2014). Se espera que individuos en estas condiciones tiendan, a su vez, a gozar de una mejor salud física (Zhong, You, Gan, Zhang, Lu \& Wang, 2009).

Por el contrario, tal y como señalan Faragher et al. (2013), cuando los individuos sienten que su trabajo no es capaz de satisfacer sus necesidades personales, tienden a sentirse infelices, insuficientemente valorados e insatisfechos. Esto suele conducir a la experimentación de distrés, ansiedad, depresión y agotamiento, elementos que contribuyen a dañar la salud mental en el mediano plazo. Asimismo, cuando estos estados negativos se prolongan de manera sostenida a través del tiempo, amenazan con perjudicar la salud física de los individuos, encontrándose asociados con diversos trastornos y aflicciones, como migrañas, dolores de espalda, tensión ocular, privación de sueño, mareos, fatiga, problemas gastrointestinales, entre otros (Nixon, Mazzola, Bauer, Krueger \& Spector, 2011).

Si bien no fue posible encontrar ninguna investigación previa publicada que haya examinado las relaciones entre la satisfacción laboral, la salud mental y la salud física en el contexto de la profesión gerencial en Argentina, con sustento en la evidencia discutida en los párrafos precedentes, se propone la siguiente hipótesis:

Hipótesis 1: La satisfacción laboral exhibirá efectos directos e indirectos sobre la salud física por medio de la salud mental. La proposición anterior se representa en la figura 1.

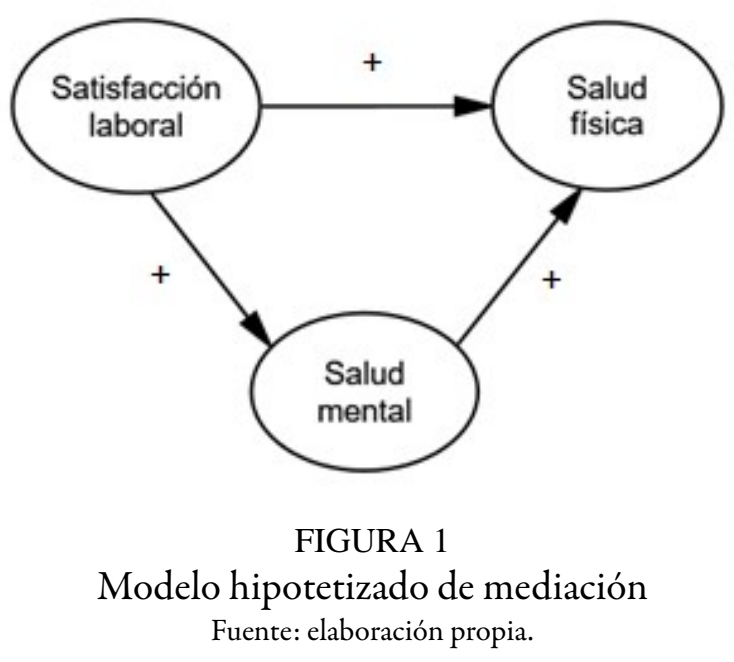

\section{METODOLOGÍA}

\section{Diseño de la investigación}

Se empleó un diseño cuantitativo, correlacional y no experimental, sustentado en una estructura de datos de corte transversal, consistente en una muestra no probabilística de gerentes argentinos. Cabe destacar que el presente artículo reporta los resultados de la primera etapa (de un total de tres) de un proyecto más amplio focalizado en el estudio del bienestar de trabajadores argentinos insertos en ocupaciones altamente demandantes. 


\section{Participantes y contexto}

La muestra se compuso por un total de 124 gerentes que, al momento de la recolección de los datos, eran estudiantes avanzados de un programa de Maestría en Administración de Negocios, dictado en modalidad part-time. Los participantes tenían desempeño en organizaciones públicas o privadas de la ciudad de Mar del Plata, la segunda ciudad más grande en términos de cantidad de habitantes de la Provincia de Buenos Aires, Argentina (ver INDEC, 2010). La muestra estaba distribuida según se describe a continuación: el 64,66\% de los participantes se encontraban en relación de dependencia en el sector privado, el 16,38\% en relación de dependencia en el sector público, y el 18,97\% ejercían el rol de consultores con una categoría equivalente o superior a senior, manifestando además ejercer la profesión de manera independiente. El 62,07\% de los participantes eran hombres y el 37,93\% mujeres. Adicionalmente, el 55,17\% estaban casados o conviviendo con su pareja y el 71,55\% trabajaban más de 40 horas semanales (full-time). La edad varió entre 26 y 65 años, con una media de 36,57 y un desvío estándar de 8,89. La totalidad de los participantes indicaron poseer personal a cargo. La antigüedad en el ejercicio de la profesión osciló entre 5 y 40 años, con una media de 11,50 y un desvío estándar de 8,03. En cuanto a la antigüedad en la organización actual, la misma varió entre 8 meses y 29 años, con una media de 8,53 y un desvío estándar de 7,49. Finalmente, la antigüedad en el cargo actual osciló entre 8 meses y 26 años, con una media aritmética de 4,93 y un desvío estándar de 4,86.

\section{Procedimiento}

En primer lugar, se solicitó al coordinador del programa su autorización para enviar a todos los cursantes de las tres cohortes activas $(N=241)$ un correo electrónico con una invitación a participar del estudio y un enlace a un cuestionario virtual, desarrollado a través de la herramienta Google Forms. La encuesta estuvo precedida por una descripción de los propósitos de la investigación y un formulario de consentimiento informado. Sólo se dio acceso a los participantes al cuestionario cuando dicho formulario fue aceptado digitalmente. Si bien las invitaciones fueron enviadas desde una dirección institucional, se aclaró a los participantes que cualquier información que permitiera identificarlos sería estrictamente confidencial, y que sólo los investigadores principales de este estudio tendrían acceso a los datos recolectados (ver Declaración de Helsinki de 1964 y Declaración de Singapur de 2010). También se detallaron en el consentimiento informado los beneficios y riesgos de participar en el estudio, y se recordó a los participantes que podrían abandonarlo en cualquier momento, sin sufrir por ello ningún tipo de consecuencia. Dado que era posible que los participantes desempeñaran más de una actividad profesional, se les solicitó que respondieran la encuesta exclusivamente desde su experiencia en su ocupación principal, la cual debía coincidir con la oportunamente declarada en la sección de datos sociodemográficos. Del total de 125 cuestionarios devueltos, uno de ellos fue eliminado debido a que presentó excesivos valores perdidos en todas las escalas, quedando la muestra final conformada por 124 participantes.

\section{Variables e instrumentos}

Satisfacción laboral. Se examinaron los componentes afectivos de la satisfacción laboral (Pujol-Cols \& Dabos, 2018) a través de una versión en español del Brief Index of Affective Job Satisfaction (BIAJS; Thompson \& Phua, 2012), validada en el contexto argentino por Pujol-Cols y Dabos (2019). Este instrumento consistió en 4 reactivos y una escala de 5 niveles $(1=$ "totalmente en desacuerdo", 5 = "totalmente de acuerdo"). Los niveles de consistencia interna de la escala resultaron adecuados $(\alpha=0,88)$.

Salud mental. La salud mental fue examinada empleando una escala ad hoc, desarrollada específicamente para los fines de esta investigación. La misma se compuso por un total de 8 reactivos (ver Tabla 1) y una 
escala estilo Likert de 6 niveles ( 1 = "nunca", 6 = "siempre". Los puntajes asignados a cada ítem fueron revertidos y re-expresados en términos positivos para que reflejen mayores niveles de salud mental. La consistencia interna de esta escala fue satisfactoria $(\alpha=0,92)$ Dado que este instrumento fue desarrollado ad hoc para esta investigación, además de analizar su consistencia interna, se examinó su estructura factorial y validez convergente, elementos que componen la validez de constructo. Los resultados del análisis factorial confirmatorio brindaron un soporte satisfactorio al modelo unidimensional de salud mental, $\chi^{2}(124,18)=20,58$, n.s., CFI $=0,997$, GFI $=0,960$, TLI $=0,995$, RMSEA $=0,034$, SRMR $=0,027$. Asimismo, la varianza media extraída (AVE se estimó en 0,64, lo que indica niveles satisfactorios de validez convergente (Fornell \& Larcker, 1981; Hair, Black, Babin, \& Anderson, 2010.

Salud fisica. La salud física fue evaluada empleando la versión en español del Physical Symptoms Inventory (PSI; Spector \& Jex, 1998, instrumento que examina el grado de presencia de 18 síntomas relacionados con la salud física durante un período de 30 días, a través de una escala compuesta por tres valores ( 3 = "nunca", 2 = "sí, pero no acudí al médico", 1 = "sí, y acudí al médico". Es importante destacar que la versión en español del PSI ya ha sido empleada previamente en el contexto argentino por Pujol-Cols (2018, donde verificó poseer propiedades psicométricas satisfactorias. En la presente investigación, la consistencia interna del PSI fue adecuada $(\alpha=0,80)$.

\section{Análisis de datos}

Con el propósito de contrastar el modelo teórico propuesto en la Figura 1, se realizó un análisis de ecuaciones estructurales en IBM SPSS Amos (versión 22). De manera previa, se verificó que los datos dieran cumplimiento al supuesto de normalidad multivariante, examinando los niveles de asimetría y curtosis multivariante por medio de la prueba de Mardia (Mardia, 1985). En la determinación de los valores críticos, se empleó el estándar de 5 sugerido por Bentler (2006). Dado que los resultados de la prueba de Mardia revelaron el incumplimiento del supuesto de normalidad multivariante, se utilizó el método bootstrap de Bollen-Stine (Bollen \& Stine, 1992) para corregir los p-valores de las pruebas $\chi^{2}$. El modelo hipotetizado incluyó una variable exógena latente (la satisfacción laboral y dos variables endógenas latentes) la salud física y la salud mental. En la especificación de los modelos, se siguieron las recomendaciones metodológicas de Bagozzi y Edwards (1998) de emplear un modelo de desagregación parcial, el cual consiste en la creación de dos o más variables observadas agregadas para cada variable latente (Dabholkar, Thorpe \& Rentz, 1996). El empleo de modelos de desagregación parcial suele ser la alternativa más apropiada cuando se cuenta con muestras pequeñas o moderadas, puesto que tiende a mejorar los índices de ajuste al reducir la cantidad de parámetros a ser estimados (Pujol-Cols \& Arraigada, 2017; Rocha \& Chelladurai, 2012). Siguiendo este enfoque, en lugar de introducir la totalidad de los reactivos de los cuestionarios como las variables observadas de cada una de las variables latentes, se construyeron parcelas para cada una de ellas (Bagozzi \& Edwards, 1998), para una descripción detallada del método, asignando los ítems a cada una de manera aleatoria (Rocha \& Chelladurai, 2012).

En la evaluación del modelo se siguieron las recomendaciones de Bollen (1989) de reportar y comparar distintos índices de ajuste, tales como el estadístico chi-cuadrado $\left(\chi^{2}\right)$, el índice comparativo de ajuste (CFI) por sus siglas en inglés, el índice de bondad del ajuste (GFI) por sus siglas en inglés, el índice de TuckerLewis (TLI) por sus siglas en inglés y el error medio cuadrático de aproximación (RMSEA) por sus siglas en inglés. Si bien no existe consenso absoluto sobre los valores críticos de cada índice de ajuste (Marsh, Hau \& Wen, 2004), de acuerdo a lo señalado por Byrne (2001), valores de CFI, TLI o GFI superiores a 0,90 y de RMSEA similares a 0,08 indican un buen ajuste a los datos. En cuanto a los resultados de la prueba chi-cuadrado, tales valores fueron interpretados con cautela al presentar este estadístico la limitación de ser muy sensible al tamaño muestral (Hooper, Coughlan \& Mullen, 2008). Finalmente, con el fin de determinar si los 
resultados del estudio daban soporte a la hipótesis de mediación, se calcularon los efectos directos, indirectos y totales; sus niveles de significatividad y los intervalos de confianza (MacKinnon, Fairchild \& Fritz, 2007; Preacher, Zhang \& Zyphur, 2011) mediante bootstrapping (1000 muestras), utilizando el método de máxima verosimilitud (Rucker, Preacher, Tormala \& Petty, 2011).

\title{
RESULTADOS
}

\section{Análisis descriptivo}

En las tablas 1 y 2 se presentan, respectivamente, los niveles reportados de salud mental y salud física. Como puede observarse en la tabla 1, más del $75 \%$ de los gerentes declararon haberse sentido estresados, cansados, tensos, nerviosos, desanimados y/o agotados mentalmente durante los 30 días anteriores a realizarse esta investigación. Además, más de un tercio del total de los gerentes reportaron haber padecido dolores de espalda, problemas de sueño, dolores de cabeza, indigestión ácida, tensión ocular y/o fatiga durante el mes previo a la realización de este estudio. Más aún, entre el 10\% y el 25\% de los gerentes declararon haber experimentado durante este lapso síntomas más severos, como trastornos estomacales, erupciones cutáneas y/ o diarrea. Sólo una proporción cercana al 10\% reportó haber experimentado los síntomas más preocupantes del listado, como problemas de respiración, dolores en el pecho, fiebre, calambres estomacales, infecciones y/ o mareos (ver tabla 2).

TABLA 1

Prevalencia de indicadores de salud mental

\begin{tabular}{|l|c|c|c|}
\hline \multirow{2}{*}{ Durante los últimos 30 días, con qué frecuencia... } & \multicolumn{3}{|c|}{ Frecuencia } \\
\cline { 2 - 4 } & Baja & Moderada & Alta \\
\hline 1. te has sentido agotado mentalmente & 20,17 & 58,87 & 20,97 \\
\hline 2. has estado cansado & 22,58 & 66,93 & 10,48 \\
\hline 3. te ha costado relajarte & 19,36 & 52,42 & 28,23 \\
\hline 4. has estado irritable & 8,88 & 50,81 & 40,32 \\
\hline 5. has estado tenso & 12,10 & 60,49 & 27,42 \\
\hline 6. te has sentido estressado & 13,71 & 56,46 & 29,84 \\
\hline 7. has estado muy nervioso & 10,49 & 37,09 & 52,42 \\
\hline 8. te has sentido tan bajo de moral que nada parecía animarte & 4,84 & 25,80 & 69,35 \\
\hline
\end{tabular}

\begin{abstract}
Notas: Frecuencia baja $=$ puntajes promedio inferiores a 2 puntos, Frecuencia moderada $=$ puntajes promedio mayores o iguales a 2 puntos y menores a 4 puntos, Frecuencia alta = puntajes promedio iguales o superiores a 4 puntos. Valores expresados en porcentajes. Fuente: elaboración propia.
\end{abstract}

TABLA 2

Prevalencia de sintomatología física

\begin{tabular}{|l|c|c|c|c|}
\hline $\begin{array}{l}\text { Durante los últimos treinta dias, presentó } \\
\text { usted... }\end{array}$ & No & Sí & $\begin{array}{c}\text { Si,y } \\
\text { acudíal } \\
\text { médico }\end{array}$ & $\begin{array}{c}\text { Presentó } \\
\text { síntoma }\end{array}$ \\
\hline 1. trastorno estomacal o nauseas & 79,03 & 17,74 & 3,23 & 20,97 \\
\hline 2. dolor de espalda (e.g. ciático, cervical) & 40,32 & 49,19 & 10,48 & 59,67 \\
\hline 3. problemas de sueño & 62,90 & 34,68 & 2,42 & 37,10 \\
\hline 4. erupción cutánea & 83,87 & 11,29 & 4,84 & 16,13 \\
\hline 5. problemas de respiración & 94,35 & 3,23 & 2,42 & 5,65 \\
\hline 6. dolor en el pecho & 91,13 & 8,87 & 0,00 & 8,87 \\
\hline 7. dolor de cabeza & 45,97 & 52,42 & 1,61 & 54,03 \\
\hline 8. fiebre & 92,74 & 4,84 & 2,42 & 7,26 \\
\hline 9. indigestión ácida o dispepsia & 73,39 & 25,00 & 1,61 & 26,61 \\
\hline 10. tensión ocular & 68,55 & 28,23 & 3,23 & 31,46 \\
\hline 11. diarrea & 88,71 & 8,06 & 3,23 & 11,29 \\
\hline 12. calambres estomacales (no menstruales) & 92,74 & 6,45 & 0,81 & 7,26 \\
\hline 13. estreñimiento & 91,94 & 5,65 & 2,42 & 8,07 \\
\hline 14. palpitaciones cardiacas & 92,74 & 6,45 & 0,81 & 7,26 \\
\hline 15. infección & 95,97 & 2,42 & 1,61 & 4,03 \\
\hline 16. pérdida de apetito & 87,90 & 11,29 & 0,81 & 12,10 \\
\hline 17. mareos & 91,94 & 6,45 & 1,61 & 8,06 \\
\hline 18. cansancio o fatiga & 47,58 & 47,58 & 4,84 & 52,42 \\
\hline
\end{tabular}

Fuente: elaboración propia. Valores expresados en porcentajes.

En la tabla 3 se presentan las medias, desviaciones estándar y correlaciones entre las variables del estudio. Como puede observarse, los niveles de satisfacción laboral, salud mental y salud física fueron, en 
promedio, moderados en la muestra. Por otro lado, la satisfacción laboral exhibió correlaciones positivas y estadísticamente significativas con la salud mental, no así con la salud física.

TABLA 3

Medias, desviaciones estándar y correlaciones entre las variables del estudio

\begin{tabular}{|l|c|c|c|c|c|}
\hline \multicolumn{1}{|c|}{ Variable } & $\mathrm{M}$ & $\mathrm{DE}$ & 1 & 2 & 3 \\
\hline 1. Satisfacción laboral & 3,75 & 0,80 & 0,88 & & \\
\hline 2. Salud mental & 4,01 & 0,91 & $0,31^{*}$ & 0,92 & \\
\hline 3. Salud fisica & 1,76 & 0,21 & 0,12 & $0,61^{*}$ & 0,80 \\
\hline
\end{tabular}

Notas: $\mathrm{M}=$ Media, $\mathrm{DE}=$ Desviación estándar, ${ }^{*} \mathrm{p}<0,0001$. Los niveles de consistencia interna (coeficiente alfa de Cronbach) se hallan reportados sobre la diagonal principal en cursivas.

Fuente: elaboración propia.

Dado que la correlación observada entre la salud mental y la salud física fue ligeramente elevada, se decidió analizar la validez discriminante entre estas dos escalas a través de un AFC, en el que se contrastaron dos modelos competitivos en Amos (para una descripción más detallada del método ver Alarcon \& Lyons, 2011; Pujol-Cols \& Lazzaro-Salazar, 2018). En el primer modelo, la salud mental y la salud física fueron ingresadas como dos variables latentes relacionadas. En el segundo, se hipotetizó que las variables observadas correspondientes a cada una saturarían en un único constructo latente. Se utilizaron las diferencias en el estadístico $\chi^{2}$ para comparar el nivel de ajuste provisto por ambos modelos. Los resultados del AFC revelaron que la caída en el estadístico $\chi^{2}$ al pasar de un modelo al otro es estadísticamente significativa $\left(\Delta \chi^{2}=110,86\right.$, $\mathrm{p}<0,0001$ ), lo que indica que el modelo que propone la salud física y la salud mental como dos factores separados, aunque relacionados, es el que brinda el mejor ajuste a los datos.

\section{Sesgo de método común}

Dado que la satisfacción laboral, la salud mental y la salud física fueron examinadas en el mismo momento temporal, situación que puede conducir al denominado sesgo de método común, se decidió realizar la prueba de un factor de Harman (Harman's one factor test). Para ello, las variables del estudio fueron simultáneamente introducidas en un análisis factorial exploratorio. Los resultados revelaron que el factor único sólo explicó el 27,54\% de la varianza total, porcentaje que es inferior al nivel crítico del 50\% señalado por Podsakoff, McKenzie, Lee \& Podsakoff (2003). Esto indica que el sesgo de método común no afectó de manera significativa los resultados del estudio.

\section{Modelo de relaciones estructurales}

Con el propósito de contrastar la hipótesis 1 se construyó un modelo de ecuaciones estructurales en Amos (versión 22). El método de estimación fue el de máxima verosimilitud. El modelo estimado incluyó una variable latente exógena (la satisfacción laboral) y dos variables latentes endógenas (la salud mental y la salud física). Los estadísticos obtenidos para el modelo hipotetizado $-\chi^{2}(11, \mathrm{~N}=124)=15,00 ; \mathrm{p}>0,10$; CFI $=0,99 ; \mathrm{GFI}=0,97$; RMSEA $=0,05 ; \mathrm{TLI}=0,98 ; \mathrm{PNFI}=0,51-$ indicaron un ajuste aceptable a los datos. Como puede observarse en la Figura 2, los resultados mostraron que aquellos directivos que experimentaron mayor satisfacción laboral tendieron a poseer estados mentales más saludables $(\beta=0,27, p<0,05)$, lo que, a su vez, se encontró relacionado con una mejor salud física $(\beta=0,73, p<0,01)$.

Con la finalidad de examinar la significatividad del efecto de mediación, se realizó la prueba de Sobel, utilizando para ello el software diseñado por Preacher y Leonardelli (2017). La hipótesis alternativa consiste en que la relación entre la variable independiente y la dependiente es un efecto indirecto que existe 
como resultado de la influencia de un mediador. Los resultados revelaron que la mediación identificada es estadísticamente significativa $(z=3,34 ; \mathrm{SE}=0,02 ; \mathrm{p}<0,0001)$.

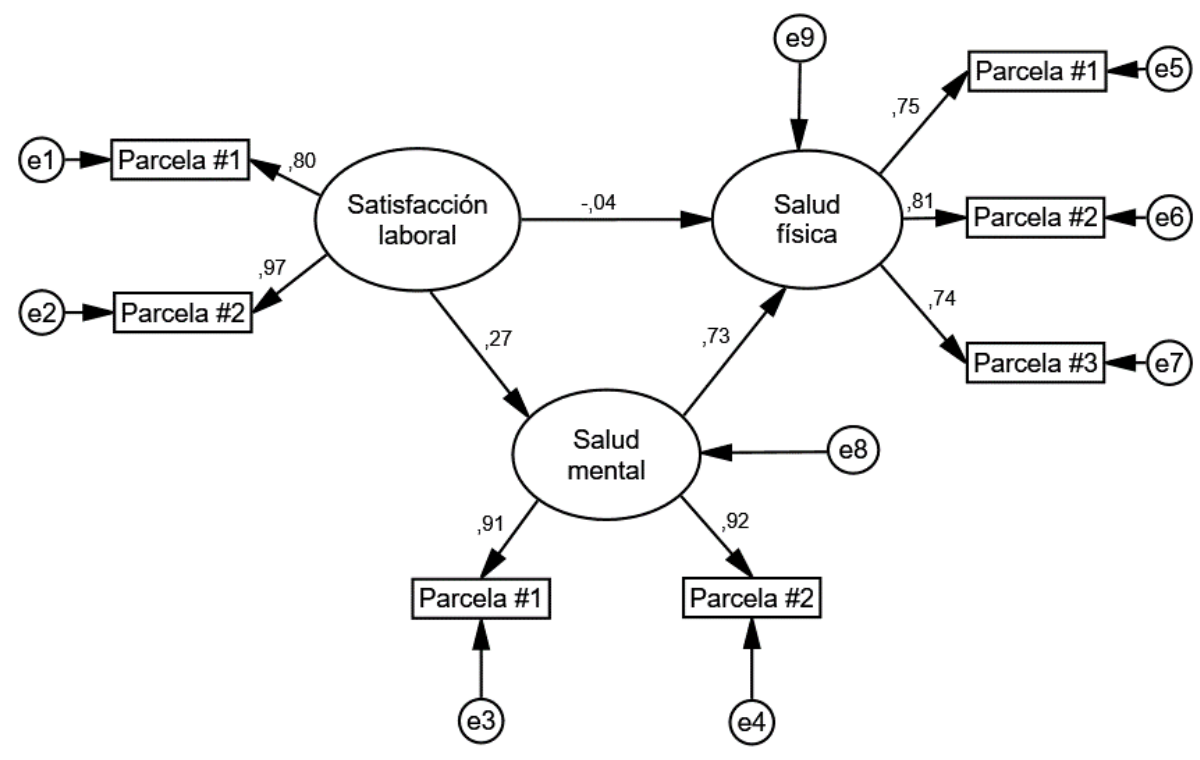

FIGURA 2

Modelo de relaciones estructurales

Fuente: elaboración propia.

Con el propósito de evaluar si los resultados del análisis de ecuaciones estructurales daban, efectivamente, soporte a la hipótesis de mediación, se procedió a calcular y analizar, mediante bootstrapping (1000 muestras) y utilizando el método de máxima verosimilitud, 1) los efectos directos, indirectos y totales, 2) sus niveles de significatividad y 3) los intervalos de confianza al 95\% (MacKinnon, Fairchild \& Fritz, 2007; Preacher, Zhang y Zyphur, 2011). El enfoque adoptado en este estudio para el análisis de las relaciones de mediación es superior al método tradicional propuesto por Baron y Kenny (1986), el cual ha sido fuertemente criticado en los últimos años (MacKinnon, 2000; MacKinnon et al., 2007).

Como puede observarse en la tabla 4, el efecto indirecto de la satisfacción laboral sobre la salud física, a través de la salud mental, fue estadísticamente significativo (coeficiente estandarizado $=0,20 ; p<0,01 ; 0,05$ $\leq 95 \% \mathrm{~B}-\mathrm{CCI} \leq 0,36)$. En cambio, los resultados indicaron que los efectos directos y totales de la satisfacción laboral sobre la salud física no fueron estadísticamente significativos.

TABLA 4

Efectos directos, indirectos y totales

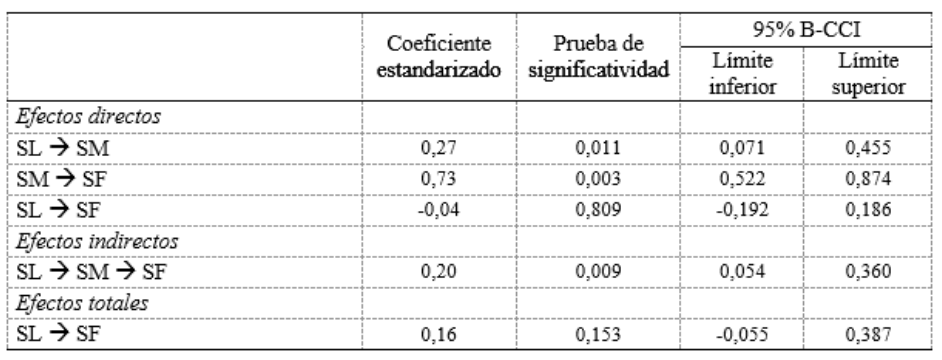

Nota. $\mathrm{N}=124 . \mathrm{SL}=$ Satisfacción laboral, $\mathrm{SM}=$ Salud mental, $\mathrm{SF}=$ Salud física, 95\% B-CCI = intervalos de confianza corregidos al 95\% Fuente: elaboración propia. 


\section{DISCUSIÓN}

La profesión gerencial constituye una de las ocupaciones más estresantes a nivel mundial. En efecto, el trabajo directivo no sólo resulta muy demandante en términos cognitivos y emocionales, sino que además suele ser desempeñado en un contexto de fuertes restricciones temporales, elevadas expectativas de comportamiento, limitado reconocimiento, insuficiente información y escaso apoyo social. Como resultado, los directivos representan un grupo profesional especialmente vulnerable a experimentar distrés, agotamiento y pérdidas de bienestar. La primera contribución de este estudio consistió en analizar la presencia de un conjunto comprehensivo de síntomas asociados con la salud física y mental en una muestra de directivos argentinos. Los niveles elevados de prevalencia sintomatológica identificados en este artículo no resultan sorprendentes en virtud de las numerosas investigaciones que han reportado niveles elevados de distrés en los directivos (Braithwaite et al., 2007; Hosie \& Sevastos, 2009; Rout, 1999). Sin embargo, nos conducen a pensar ¿hasta qué punto las organizaciones pueden pretender que directivos en estas condiciones alcancen metas desafiantes y, al mismo tiempo, sean capaces de construir ambientes saludables de trabajo que motiven y retengan al personal bajo su dirección?

La segunda contribución de esta investigación consistió en analizar los efectos directos e indirectos de la satisfacción laboral sobre la salud de los directivos, por medio de un modelo de ecuaciones estructurales. Específicamente, los resultados revelaron que la satisfacción laboral no pareciera afectar la salud física de los participantes de manera directa, sino de modo indirecto, por medio de su impacto en la salud mental. En este sentido, los hallazgos de esta investigación demuestran que el trabajo pareciera asumir un rol protector en el bienestar de los individuos cuando es capaz de brindarles oportunidades sufi cientes para satisfacer sus necesidades personales, generando así satisfacción laboral. Esto es consistente con lo observado por Satuf et al. (2018) en el contexto europeo. Así, los resultados reportados en este estudio no sólo brindan soporte a investigaciones previas, realizadas en otros contextos ocupacionales, en las que se reportan relaciones positivas entre la satisfacción laboral y la salud (Faragher et al., 2013; Grant et al., 2009; Pujol-Cols, 2018; Tsaousis et al., 2007), sino que además contribuyen a mejorar el entendimiento acerca de los mecanismos que subyacen a la vinculación entre estos fenómenos.

La no verificación de efectos directos de la satisfacción laboral sobre la salud física resulta razonable, puesto que el distrés suele demorar un cierto lapso de tiempo hasta que se manifiesta en una pérdida de salud física (Pindek et al., 2018). Dado que el impacto del distrés a nivel sintomatológico suele darse en el mediano o largo plazo, es probable que por este motivo las correlaciones reportadas, no sólo en este estudio sino también en investigaciones previas, entre la satisfacción laboral y la salud física, sean más débiles que las observadas entre la satisfacción laboral y la salud mental (ver Faragher et al., 2013). Al respecto, como señalan Rucker, Preacher, Tormala y Petty (2011), la no detección de efectos directos y/o totales no excluye la posibilidad de observar efectos indirectos, sino que los segundos pueden existir aún frente a la ausencia de los primeros. De hecho, "esta situación suele ser bastante frecuente - ocurre casi en la mitad de los casos - frente a los tamaños muestrales con los que cuentan típicamente las investigaciones en psicología social" (Rucker et al., 2011, p. 362, traducción de los autores).

Tradicionalmente, la literatura organizacional ha señalado que los rasgos de personalidad asumen un rol fundamental en el modo en que los directivos perciben, experimentan y responden al estrés (PujolCols \& Dabos, 2018). Específicamente, se ha demostrado que los directivos tienden a poseer rasgos de personalidad muy positivos, como lo son la autoestima, la autoeficacia, el locus interno de control y la estabilidad emocional. Estos rasgos, a su vez, les permiten focalizarse en los aspectos más positivos de su trabajo, percibir los eventos como menos estresantes, tener mayor confianza en su capacidad para alcanzar las metas de manera exitosa, ser más efectivos en la resolución de los problemas y, como resultado, experimentar menores niveles de distrés durante el ejercicio de su rol. Sin embargo, esto nos lleva a pensar: ¿hasta qué punto es ético que las políticas de gestión de personal recaigan exclusivamente en la posesión de estos rasgos positivos de personalidad? 
¿No podría ser ésta una excusa para no introducir modificaciones en el diseño del trabajo que permitan a los directivos afrontar más saludablemente los estresores laborales? ¿En qué momento estos rasgos positivos dejarán de ser suficientes para afrontar las demandas de la profesión y se generarán consecuencias graves en la salud física y mental de los directivos? Desde luego, los interrogantes anteriores aguardan mayores esfuerzos de investigación.

Si bien es cierto que muchas de las demandas enfrentadas diariamente por los gerentes durante el ejercicio de su rol son intrínsecas de su profesión, lo que significa que son poco modificables por la organización, estas últimas sí poseen la capacidad para introducir cambios en algunas de las condiciones en las que los directivos desempeñan sus tareas. En este sentido, al tratarse de una profesión tan estresante, las organizaciones podrían mitigar su impacto psicosocial sobre la salud de los directivos brindándoles los recursos que les permitan enfrentar con mayor efectividad las demandas del trabajo (ver para este punto las proposiciones de la teoría de demandas y recursos laborales en Bakker y Demerouti, 2014). Dentro de los recursos aludidos, las organizaciones podrían realizar las siguientes acciones: conceder mayor autonomía para realizar el trabajo, estableciendo controles amplios y basados en el desempeño; diseñar planes de carrera que permitan el crecimiento y el aprendizaje continuo; brindar apoyo y acompañamiento frente a situaciones difíciles de resolver; mantener abiertas las vías de comunicación tanto en sentido descendente como ascendente; ofrecer condiciones flexibles de trabajo, especialmente a aquellos que poseen cargas de familia, entre otros. Estas acciones, además de contribuir a incrementar la satisfacción laboral, permitirían que los directivos desarrollen su capital psicológico positivo, lo que no sólo mejoraría su efectividad, sino también su capacidad para enfrentar los estresores laborales (Luthans, Norman, Avolio \& Avey, 2008; Gooty, Gavin, Johnson, Frazier \& Snow, 2009). Se concluye que es esencial que las organizaciones inviertan recursos en mejorar las condiciones laborales que ofrecen a sus gerentes, con el propósito de acrecentar su satisfacción laboral y, de este modo, preservar su salud física y mental.

Se finaliza esta sección reconociendo las principales limitaciones de este estudio y proponiendo líneas futuras de investigación. En primer lugar, este estudio se concentró en las relaciones entre la satisfacción laboral y la salud. Futuras investigaciones podrían ampliar el modelo propuesto incorporando medidas perceptuales y objetivas de recursos y demandas laborales, además de otras relativas a la personalidad de los individuos. En segundo lugar, el modelo elaborado fue contrastado empíricamente en una muestra pequeña y, en gran medida, homogénea, lo que compromete la generalización de los hallazgos. Futuras investigaciones podrían contrastar el modelo propuesto en muestras más grandes y heterogéneas de trabajadores. En tercer lugar, la muestra utilizada en esta investigación fue de naturaleza no probabilística, por lo que no es posible afirmar que las percepciones de quienes respondieron el cuestionario son equivalentes a las de quienes no lo hicieron. En cuarto lugar, en esta investigación no fue posible controlar los efectos examinados por el nivel organizacional en el que se desempeñaban los directivos participantes. Dado que las demandas y recursos laborales pueden ser diferentes según se trate de gerentes de nivel medio o superior, futuras investigaciones deberían controlar los efectos examinados en este estudio en función de la jerarquía. En quinto lugar, este estudio se fundamentó en datos de corte transversal, lo que impide a los autores postular relaciones de causalidad entre las variables. Así, del mismo modo en que se señaló que niveles más elevados de satisfacción laboral tienden a conducir a estados mentales más saludables, la relación inversa resulta igualmente válida (esto es, aquellos sujetos más sanos mentalmente podrían ser más proclives a experimentar actitudes más positivas hacia el trabajo). Futuras investigaciones podrían solucionar el inconveniente de la causalidad empleando diseños longitudinales, que procuren mediciones sistemáticas de las variables de interés a través del tiempo. Finalmente, las medidas de salud física y mental utilizadas en este estudio fueron exclusivamente perceptuales. Se cree fundamental que futuras investigaciones triangulen la evidencia obtenida a través de escalas de autoreporte con la que surja de diagnósticos médicos y entrevistas clínicas. Esto último favorecería, a su vez, la interdisciplinariedad, la cual resulta esencial para comprender más cabalmente los procesos implicados en la experimentación de estados saludables en el trabajo. 


\section{REFERENCIAS}

Alarcon, G. M., \& Lyons, J. B. (2011). The relationship of engagement and job satisfaction in working samples. The Journal of Psychology, 145, 463-480. https://doi.org/10.1080/00223980.2011.584083

Bagozzi, R., \& Edwards, J. (1998). A general approach for representing constructs in organizational research. Organizational Research Methods, 1(1), 45-87. https://doi.org/10.1177/109442819800100104

Bakker, A. \& Demerouti, E. (2014). Job demands-resources theory. In C. Cooper \& P. Chen (eds.), Wellbeing. $A$ Complete reference guide (pp. 37-64). Chichester: Wiley-Blackwell.

Baron, R., \& Kenny, D. (1986). The moderator-mediator variable distinction in social psychological research: Conceptual, strategic, and statistical considerations. Journal of Personality and Social Psychology, 51(6), 1173-1182. https://doi.org/10.1037//0022-3514.51.6.1173

Bentler, P. (2006). EQS 6 structural equations program manual. Encino, CA: Multivariate Software Inc.

Birnberg, J., \& Luft, J. (2007). Psychology theory in management accounting research. In C. Chapman, A. Hopwood, \& M. Shields (eds.), Handbook of Management Accounting Research. Oxford: Elsevier.

Bollen, K. (1989). A new incremental fit index for general structural equation models. Sociological Methods and Research, 17(3), 303-316. https://doi.org/10.1177/0049124189017003004

Bollen, K., \& Stine, R. (1992). Bootstrapping goodness-of-fit measures in structural equation models. Sociological Methods \& Research, 21(2), 205-229. https://doi.org/10.1177/0049124192021002004

Bowling, N., Alarcon, G., Bragg, C., \& Hartman, M. (2015). A meta-analytic examination of the potential correlates and consequences of workload. Work \& Stress, 29(2), 95-113. https://doi.org/10.1080/02678373.2015.1033 037

Bowling, N., Eschleman, K., \& Wang, Q. (2010). A meta-analytic examination of the relationship between job satisfaction and subjective well-being. Journal of Occupational and Organizational Psychology, 83(4), 915-934. h ttps://doi.org/10.1348/096317909x478557

Braithwaite, J., Westbrook, M., \& Mallock, N. (2007). Pressures exerted on managers by their superior and peer managers: Australian-Singaporean comparisons. Journal of Managerial Psychology, 22(3), 227-256. https://doi .org/10.1108/02683940710733070

Brotheridge, C., \& Grandey, A. (2002). Emotional labor and burnout: Comparing two perspectives of "people work". Journal of Vocational Behavior, 60(1), 17-39. https://doi.org/10.1006/jvbe.2001.1815

Collinson, D., \& Collinson, M. (1997). Delayering managers': Time-space surveillance and its gendered effects. Organization, 4(3), 375-407. https://doi.org/10.1177/135050849743005

Christie, A., \& Barling, J. (2009). Disentangling the indirect links between socioeconomic status and health: The dynamic roles of work stressors and personal control. Journal of Applied Psychology, 94(6), 1466-1478. https:// doi.org/10.1037/a0016847

Dabos, G. E., \& Rivero, A. G. (2012). Contratos idiosincrásicos en la atracción y retención del talento: tres estudios en organizaciones intensivas en conocimiento de la Argentina. Estudios Gerenciales, 28(125), 3-12. https://doi .org/10.1016/s0123-5923(12)70002-5

Dabholkar, P., Thorpe, D. \& Rentz, J. (1996). A measure of service quality for retail stores: scale development and validation. Journal of the Academy of Marketing Science, 24(1), 3-16. https://doi.org/10.1007/bf02893933

Diener, E., \& Tay, L. (2017). A scientific review of the remarkable benefits of happiness for successful and healthy living. In The Centre for Bhutan Studies, Happiness: Transforming the Development Landscape (pp. 90-117). Bhutan: The Centre for Bhutan Studies and GNH.

Diestel, S., Wegge, J., \& Schmidt, K. (2014). The impact of social context on the relationship between individual job satisfaction and absenteeism: The roles of different foci of job satisfaction and work-unit absenteeism. Academy of Management Journal, 57(2), 353-382. https://doi.org/10.5465/amj.2010.1087 
Downey, L. A., Papageorgiou, V., \& Stough, C. (2006). Examining the relationship between leadership, emotional intelligence and intuition in senior female managers. Leadership \& Organization Development Journal, 27(4), 250-264. https://doi.org/10.1108/01437730610666019

Faragher, E. B., Cass, M., \& Cooper, C. L. (2013). The relationship between job satisfaction and health: a meta-analysis. In C. Cooper (ed.), From Stress to Wellbeing Volume 1 (pp. 254-271). London: Palgrave Macmillan.

Flickinger, M., Allscher, M., \& Fiedler, M. (2016). The mediating role of leader-member exchange: A study of job satisfaction and turnover intentions in temporary work. Human Resource Management Journal, 26(1), 46-62. h ttps://doi.org/10.1111/1748-8583.12091

Forbes (2017). The 10 Most Stressful Jobs in 2017 [Blog post]. Retrieved from: https://www.forbes.com/pictures/fe ki45eidhi/the-10-most-stressful-jo/\#3655fdc5437f

Ford, J., \& Collinson, D. (2011). In search of the perfect manager? Work-life balance and managerial work. Work, Employment and Society, 25(2), 257-273. https://doi.org/10.1177/0950017011398895

Gardner, L., \& Stough, C. (2002). Examining the relationship between leadership and emotional intelligence in senior level managers. Leadership \& Organization Development Journal, 23(2), 68-78. https://doi.org/10.1108/0143 7730210419198

Gooty, J., Gavin, M., Johnson, P., Frazier, M., \& Snow, D. (2009). In the eyes of the beholder: Transformational leadership, positive psychological capital, and performance. Journal of Leadership \& Organizational Studies, 15(4), 353-367. https://doi.org/10.1177/1548051809332021

Grant, N., Wardle, J., \& Steptoe, A. (2009). The relationship between life satisfaction and health behavior: a crosscultural analysis of young adults. International Journal of Behavioral Medicine, 16(3), 259-268. https://doi.org /10.1007/s12529-009-9032-x

Hall, M. (2010). Accounting information and managerial work. Accounting, Organizations and Society, 35(3), 301-315. https://doi.org/10.1016/j.aos.2009.09.003

Hentrich, S., Zimber, A., Sosnowsky-Waschek, N., Gregersen, S. \& Petermann, F. (2017). The role of core selfevaluations in explaining depression and work engagement among managers. Current Psychology, 36(3), 516-529. https://doi.org/10.1007/s12144-016-9439-x

Hooper, D., Coughlan, J., \& Mullen, M. (2008). Structural Equation Modelling: Guidelines for Determining Model Fit. Electronic Journal of Business Research Methods, 6(1), 53-60.

Hosie, P., \& Sevastos, P. (2009). Does the "happy-productive worker" thesis apply to managers? International Journal of Workplace Health Management, 2(2), 131-160. https://doi.org/10.1108/17538350910970219

INDEC (2010). Censo 2010: Cuadros definitivos - Buenos Aires Interior. Retrieved from: https://www.indec.gov.a $\mathrm{r} / \mathrm{ftp} / \mathrm{censos} / 2010 /$ CuadrosDefinitivos/P1-P_Buenos_Aires_Interior.pdf

Lewig, K., \& Dollard, M. (2003). Emotional dissonance, emotional exhaustion and job satisfaction in call centre workers. European Journal of Work and Organizational Psychology, 12(4), 366-392. https://doi.org/10.1080/1 3594320344000200

Lucia-Casademunt, A., Ariza-Montes, J., \& Morales-Gutiérrez, A. (2013). Determinants of occupational well-being among executive women. Revista Latinoamericana de Administración, 26(2), 229-257. https://doi.org/10.110 8/arla-06-2013-0064

Luft, J., \& Shields, M. (2003). Mapping management accounting: graphics and guidelines for theory-consistent empirical research. Accounting, Organizations and Society, 28(2-3), 169-249. https://doi.org/10.1016/s0361-3 682(02)00026-0

Luthans, F., Norman, S., Avolio, B., \& Avey, J. (2008). The mediating role of psychological capital in the supportive organizational climate-employee performance relationship. Journal of Organizational Behavior, 29(2), 219-238. https://doi.org/10.1002/job.507

MacKinnon, D. P. (2000). Contrasts in multiple mediator models. In J. Rose, L. Chassin, C. Presson, \& S. Sherman (eds.), Multivariate applications in substance use research: New methods for new questions (pp. 141-160). New York: Lawrence Erlbaum Associates.

MacKinnon, D., Fairchild, A., \& Fritz, M. (2007). Mediation analysis. Annual Review in Psychology, 58(1), 593-614. 
Manshor, A., Fontaine, R., \& Siong Choy, C. (2003). Occupational stress among managers: a Malaysian survey.Journal of Managerial Psychology, 18(6), 622-628. https://doi.org/10.1108/02683940310494412

Mardia, K. (1985). Mardia's test of multinormality. In S. Kotz \& N. Johnson (eds.), Encyclopedia of Statistical Sciences (pp. 217-221). New York: Wiley.

Mark, G., Gonzalez, V., \& Harris, J. (2005, April). No task left behind? Examining the nature of fragmented work. In Proceedings of the SIGCHI conference on Human factors in computing systems (pp. 321-330), Portland, Oregon.

Marsh, H., Hau, K., \& Wen, Z. (2004). In search of golden rules: Comment on hypothesis-testing approaches to setting cutoff values for fit indexes and dangers in overgeneralizing Hu and Bentler's (1999) findings. Structural Equation Modeling, 11(3), 320-341. https://doi.org/10.1207/s15328007sem1103_2

Mercado Salgado, P. \& Salgado Mejía, R. (2008). Estrés en ejecutivos de medianas y grandes empresas mexicanas: un enfoque de desarrollo humano organizacional. Estudios Gerenciales, 24(108), 15-36. https://doi.org/10.1016/ s0123-5923(08)70042-1

Mohr, G., \& Wolfram, H. (2010). Stress among managers: The importance of dynamic tasks, predictability, and social support in unpredictable times. Journal of Occupational Health Psychology, 15(2), 167-179. https://doi.org/10 $.1037 / \mathrm{a} 0018892$

Montgomery, A., Peeters, M., Schaufeli, W., \& Ouden, M. (2003). Work-home interference among newspaper managers: Its relationship with burnout and engagement. Anxiety, Stress, and Coping, 16(2), 195-211. https:// doi.org/10.1080/1061580021000030535

Nixon, A., Mazzola, J., Bauer, J., Krueger, J., \& Spector, P. (2011). Can work make you sick? A meta-analysis of the relationships between job stressors and physical symptoms. Work \& Stress, 25(1), 1-22. https://doi.org/10.108 $0 / 02678373.2011 .569175$

O’Neill, J. W., \& Xiao, Q. (2010). Effects of organizational/occupational characteristics and personality traits on hotel manager emotional exhaustion. International Journal of Hospitality Management, 29(4), 652-658. https://doi. org/10.1016/j.ijhm.2009.12.004

Pindek, S., Arvan, M., \& Spector, P. (2018). The stressor-strain relationship in diary studies: A meta-analysis of the within and between levels. Work \& Stress, 1-21. https://doi.org/10.1080/02678373.2018.1445672

Preacher, K. J., Zhang, Z., \& Zyphur, M. J. (2011). Alternative methods for assessing mediation in multilevel data: The advantages of multilevel SEM. Structural Equation Modeling, 18(2), 161-182.

Preacher, K. J., \& Leonardelli, G. J. (2017). Calculation for the Sobel test: An interactive calculation tool for mediation tests. Disponible en http://quantpsy.org/sobel/sobel.htm

Pujol-Cols, L., \& Arraigada, M. C. (2017). Propiedades psicométricas del Cuestionario de Riesgos Psicosociales Copsoq-Istas 21 y aplicación en docentes universitarios argentinos. Cuadernos de Administración, 30(55), 97-125. https://doi.org/10.11144/javeriana.cao30-55.ppcr

Pujol-Cols, L., \& Lazzaro-Salazar, M. (2018). Psychosocial Risks and Job Satisfaction in Argentinian Scholars: Exploring the Moderating Role of Work Engagement. Journal of Work and Organizational Psychology, 34(3), 145-156. https://doi.org/10.5093/jwop2018a17

Pujol-Cols, L, \& Dabos, G. (2019). Dispositional and situational factors at work: A validation of scales and examination of effects on job satisfaction. Academia Revista Latinoamericana de Administración. https://doi.o $\mathrm{rg} / 10.1108 /$ arla-12-2017-0355

Pujol-Cols, L., \& Dabos, G. (2018). Satisfacción laboral: una revisión de la literatura acerca de sus principales determinantes. Estudios Gerenciales, 34(146), 3-18. https://doi.org/10.18046/j.estger.2018.146.2809

Pujol-Cols, L. (2018). Work engagement, satisfacción laboral, salud física y salud mental en académicos de una universidad pública argentina. Salud de los Trabajadores, 26(1), 6-19.

Pujol-Cols, L., \& Arraigada, M. (2018). Propiedades psicométricas de la versión corta de la Utrecht Work Engagement Scale en trabajadores argentinos. Pensamiento Psicológico, 16(2), 31-45. https://doi.org/10.11144/javerianacal i.ppsi16-2.ppvc 
Rocha, C. \& Chelladurai, P. (2012). Item parcels in structural equation modeling: An applied study in sport management. International Journal of Psychology and Behavioral Sciences, 2(1), 46-53. https://doi.org/10.5923 /j.ijpbs.20120201.07

Ridner, S. (2004). Psychological distress: Concept analysis. Journal of Advanced Nursing, 45(5), 536-545. https://do i.org/10.1046/j.1365-2648.2003.02938.x

Rout, U. (1999). Job stress among general practitioners and nurses in primary care in England. Psychological Reports, 85(3), 981-986. https://doi.org/10.2466/pr0.85.7.981-986

Rucker, D., Preacher, K., Tormala, Z., \& Petty, R. (2011). Mediation analysis in social psychology: Current practices and new recommendations. Social and Personality Psychology Compass, 5(6), 359-371.

Salanova, M., Martínez, I., \& Llorens, S. (2014). Una mirada más “positiva” a la salud ocupacional desde la psicología organizacional positiva en tiempos de crisis: aportaciones desde el equipo de investigación WoNT. Papeles del Psicólogo, 35(1), 22-30.

Satuf, C., Monteiro, S., Pereira, H., Esgalhado, G., Marina Afonso, R., \& Loureiro, M. (2018). The protective effect of job satisfaction in health, happiness, well-being and self-esteem. International Journal of Occupational Safety and Ergonomics, 24(2), 181-189. https://doi.org/10.1080/10803548.2016.1216365

Schaufeli, W. B., Salanova, M., González-Romá, V., \& Bakker, A. B. (2002). The measurement of engagement and burnout: A two sample confirmatory factor analytic approach. Journal of Happiness Studies, 3(1), 71-92. https ://doi.org/10.1023/a:1015630930326

Seligman, M. \& Csikszentmihalyi, M. (2000). Positive psychology: An introduction. American Psychologist, 55(1), 5-14. https://doi.org/10.1037//0003-066x.55.1.5

Siegrist, J. (2016). Stress in the workplace. En Cockerham, W. (ed.), The New Blackwell Companion to Medical Sociology. Oxford: John Wiley y Sons.

Spector, P., Chen, P., \& O'Connell, B. (2000). A longitudinal study of relations between job stressors and job strains while controlling for prior negative affectivity and strains. Journal of Applied Psychology, 85(2), 211-218. https: //doi.org/10.1037//0021-9010.85.2.211

Spector, P., \& Jex, S. (1998). Development of four self-report measures of job stressors and strain: Interpersonal conflict at work scale, organizational constraints scale, quantitative workload inventory, and physical symptoms inventory. Journal of Occupational and Health Psychology, 3(4), 356-367. https://doi.org/10.1037//1076-8998 .3 .4 .356

Sprinkle, G. B. (2003). Perspectives on experimental research in managerial accounting. Accounting, Organizations and Society, 28(2-3), 287-318. https://doi.org/10.1016/s0361-3682(01)00058-7

Thompson, E. R., \& Phua, F. T. (2012). A brief index of affective job satisfaction. Group \& Organization Management, 37(3), 275-307. https://doi.org/10.1177/1059601111434201

Tsaousis, I., Nikolaou, I., Serdaris, N., \& Judge, T. (2007). Do the core self-evaluations moderate the relationship between subjective well-being and physical and psychological health?. Personality and Individual Differences, 42(8), 1441-1452. https://doi.org/10.1016/j.paid.2006.10.025

Tziner, A., Waismal-Manor, R., Vardi, N., \& Brodman, A. (2008). The personality dispositional approach to job satisfaction and organizational commitment. Psychological Reports, 103(2), 435-442. https://doi.org/10.2466/ pr0.103.2.435-442

Unanue, W., Gómez, M., Cortez, D., Oyanedel, J., \& Mendiburo-Seguel, A. (2017). Revisiting the link between job satisfaction and life satisfaction: The role of basic psychological needs. Frontiers in Psychology, 8, 1-17. https:// doi.org/10.3389/fpsyg.2017.00680

Unda, S., Uribe, F., Jurado, S., García, M., Tovalín, H., \& Juárez, A. (2016). Elaboración de una escala para valorar los factores de riesgo psicosocial en el trabajo de profesores universitarios. Revista de Psicología del Trabajo y de las Organizaciones, 32(2), 67-74. https://doi.org/10.1016/j.rpto.2016.04.004

Weir, K. (2013, December). More than job satisfaction. APA Monitor on Psychology, 44(11), 39. 
Ziegler, R., Hagen, B., \& Diehl, M. (2012). Relationship between job satisfaction and job performance: Job ambivalence as a moderator. Journal of Applied Social Psychology, 42(8), 2019-2040. https://doi.org/10.1111/j $.1559-1816.2012 .00929 . x$

Zhong, J., You, J., Gan, Y., Zhang, Y., Lu, C., \& Wang, H. (2009). Job stress, burnout, depression symptoms, and physical health among Chinese university teachers. Psychological Reports, 105(3), 1248-1254.

\section{Notas}

* Artículo de investigación.

\section{Licencia Creative Commons CC BY 4.0}

Cómo citar: Pujol-Cols, L., y Foutel, M. (2019). Satisfacción laboral y salud: Un análisis de efectos directos e indirectos en gerentes argentinos. Cuadernos de Administración, 32(59). DOI: https://doi.org/10.11144 /Javeriana.cao32-59.slsaed 